\title{
Research on Prediction Model of Improved BP Neural Network Optimized by Genetic Algorithm
}

\author{
Anzhi Qi ${ }^{1, a}$ \\ ${ }^{1}$ Liaoning Jianzhu Vocational College, Liaoyang, 111000, China \\ ${ }^{\mathrm{a}}$ email
}

Keywords: Prediction model, Improved BP neural network, Genetic algorithm

\begin{abstract}
Artificial neural networks have been applied in many fields, and with the continuous improvement of neural networks and the combination of other new algorithms, the application fields of artificial neural networks have become wider and wider. In this paper, genetic algorithm is used to optimize the performance of BP neural network. The numerical simulation results show that the BP neural network optimized by genetic algorithm can effectively avoid the local minimum defect of the original BP neural network, and has the advantages of fast convergence speed and high accuracy.
\end{abstract}

\section{Introduction}

Nowadays, wind power mainly uses near surface wind energy. Near surface wind has the characteristics of fluctuation, intermittence, low energy density and so on, so the wind power is fluctuant. When large scale wind farms are connected to the grid, large fluctuations in wind power will adversely affect the power balance and frequency regulation of the grid. How to accurately predict the power of wind farm, to provide a pre-arranged schedule the theoretical foundation for the electric power dispatching department, to ensure the safe operation of power grid and power balance is an urgent need to solve the problem. Wind energy, as one of the renewable energy sources, has the characteristics of non-pollution and transferability. How to make its value further improved to become one of the important research topics in twenty-first century. When other countries in the world have made further research, and achieved initial results and economic effects, the Chinese government has also increased the importance of renewable and clean energy. With the promulgation and implementation of "renewable energy law", China has also obtained rapid development in this field, especially in the mature technology, business prospects are optimistic about wind power generation has become the first other kinds of renewable energy. Vigorously develop renewable energy is also an important part of our energy development strategy. Wind power generation is a clean renewable energy, which can partly replace coal-fired power generation capacity and reduce pollutant emissions. At the same time, wind energy resources are very rich in our country, with the rapid development of China's wind power, wind energy resources in China is rich in wind power, the rapid development of land, wind power as a development cost relatively small green energy will gradually become a form of power supply cannot be ignored, and supported by the strong national policy. In the electricity market, wind farms predict wind power and participate in power market bidding. Power Grid Corp forecasts wind power to ensure safe and economic operation of the system.

\section{Improved BP Neural Network Optimized by Genetic Algorithm}

Summary of Neural Network. In data analysis, prediction is often used, but the traditional forecasting methods have great limitations when they involve many variables and irregular data. The emergence of neural networks provides a new approach to deal with nonlinear problems. Because the neural network has large-scale parallel and distributed storage and processing, self-organizing, adaptive and self-learning ability, especially suitable for processing the need to consider many factors and conditions are not accurate, the problem of fuzzy information processing. Artificial neural networks, also referred to as neural networks, originated in 1940s. It is a kind of neural network model of animal behavior characteristics, highly complex nonlinear network system consists of many 
simple neurons which are connected, through the adjustment of the internal relationship between the number of nodes connected to each other to achieve the purpose of processing information. Because of its inherent strong adaptive ability and learning ability, neural network has been widely used in many fields, solved many problems that traditional methods are difficult to solve, and play a huge role. It has played an important role in artificial intelligence, automatic control, communication engineering, fuzzy recognition and so on. Artificial neuron is the information processing unit in neural network, and the processing of information is nonlinear. When in use, the available mathematical model to reflect the characteristics and function of neural traditional ethics sampling.

BP Neural Network. BP neural network is a multilayer feedforward neural network. The name is derived from the adjustment rules of network weights, which uses the back-propagation learning algorithm, namely the error back propagation algorithm, called the BP learning algorithm. According to statistics, most of the neural network models adopt the BP network or its change form. The sample is provided to the network, and the size of the error $\mathrm{E}$ is preset according to the training of the above process. When the difference between the training result and the target value is less than the preset error limit, the network converges and the training ends. If the number of training has reached the maximum number of training times, training terminated, the network cannot converge. End of learning process. The error propagation process is reversed, and is trained continuously according to the error feedback until the condition is satisfied or trained to the maximum number of stops. Although the BP network has been widely used, but there are many defects, because the neural network established itself is a process of trial, the BP network layer and the number of nodes in each layer are constantly trying to improve. In addition, for the learning process of neural network, it has already formed a lot of learning algorithms, but these algorithms in mathematical calculations are more complex, the process is tedious, error prone, according to the BP neural network, and the learning rate is invariant, caused by the network convergence speed is slow, the training time is lengthened; if the problem is complicated, the training time required may be very long. An improved method is often used, such as variable learning rate or adaptive learning rate. BP training is divided into two types: single sample training and batch training. In practice, batch training is used to train the network. When the actual output is inconsistent with the expected output, the backward propagation stage of error is entered. Through the network, the error signal is returned along the original connection path, and the weights of each layer neuron are modified until the desired target is reached. Information forward and back propagation process again and again, is a process of constantly adjust the weights, and the neural network training process, this process has been to reduce the errors of network output to an acceptable level, or the number of preset learning stops.

Improved BP Neural Network Optimized by Genetic Algorithm. Although the BP neural network has high accuracy, there are still some defects. For example, when the initial value is not appropriate, it has some errors, but the topological structure is not comprehensive. Based on the above neural network defects analysis, we use genetic algorithm to improve the BP neural network, so that its accuracy is higher. The basic idea of the improved neural network with genetic algorithm firstly by genetic algorithm structure of neural network, the initial connection weights, optimize the design of the initial threshold and learning rate and momentum factor, locate the better search space in the solution space, then the BP algorithm is used in the small solution space of the network connection weights and thresholds re optimization, search the optimal solution, the optimized BP neural network can better predict the output function. The elements of BP neural network optimized by genetic algorithm include population initialization, fitness function, selection operation, crossover operation and mutation operation. Genetic algorithm is a global optimization algorithm consisting of three basic operators, selection operator, crossover operator and mutation operator, which are generated by simulating biological evolution. It starts with an initial family, selects the male parent with good character by selection operator, performs crossover operation by crossover operator, makes a little variation of mutation operator, and searches the model space randomly under certain rule of probability control. From generation to generation, the error functional values of the solution family meet the set requirements. Usually, the neural network connection weights and threshold and learning rate and momentum factor limiting the range of variation, so the connection weights and 
thresholds and the learning rate and momentum factor value represented by the string as the actual value:

$$
w=w_{\min }+\frac{b i n}{2^{N}-1}\left[w_{\max }-w_{\min }\right]
$$

The decimal integer value bin represented by a bit binary string; $\left[w_{\max }, w_{\min }\right]$ the weight and threshold of each connection, and the range of learning rate and momentum factor.

\section{Establishment of Prediction Model of Improved BP Neural Network Optimized by Genetic Algorithm}

Algorithm Process. The individual encoding is a real number encoding, and everyone is a real string, which consists of 4 parts: the input layer and the hidden layer, the hidden layer threshold, the hidden layer and the output layer connection weight and the output layer threshold. The individual contains all the weights and thresholds of the neural network. Under the condition that the network structure is known, it can form a neural network whose structure, weight and threshold are determined.

According to the individual BP neural network initial weights and thresholds, predict the system output using the training data to train the BP neural network, the prediction error between the output and the expected output of the absolute value and $\mathrm{E}$ as fitness of $\mathrm{F}$, the calculation formula is as follows:

$$
F=k\left(\sum_{i=1}^{n} a b s\left(y_{i}-o_{i}\right)\right)
$$

The $\mathrm{n}$ is the output node number of the network; yi is the expected output of the i node of the BP neural network; oi is the predicted output of the i node; $\mathrm{k}$ is the coefficient.

The genetic algorithm has many options such as roulette, tournament and so on. This paper chooses roulette method, that is, the selection strategy based on fitness ratio, and the selection probability of each individual pi is:

$$
\begin{aligned}
& f_{i}=k / F_{i} \\
& p_{i}=\frac{f_{i}}{\sum_{j=1}^{N} f_{j}}
\end{aligned}
$$

In the formula, $\mathrm{Fi}$ is the fitness value of individual $\mathrm{I}$, and the smaller the fitness value is, the better is the reciprocal of fitness value before the individual selection; $\mathrm{K}$ is the coefficient; $\mathrm{N}$ is the individual number of the population.

Because the individual uses real number encoding, the crossover operation adopts the real number crossing method, and the cross-operation method of the k chromosome ak and the l chromosome ai in the $\mathrm{j}$ bit is as follows:

$$
a_{i j}=\left\{\begin{array}{l}
a_{i j}+\left(a_{i j}-a_{\max }\right) \times f(g), r>0.5 \\
a_{i j}+\left(a_{\text {min }}-a_{i j}\right) \times f(g), r \leq 0.5
\end{array}\right.
$$

BP neural network genetic algorithm extremum optimization mainly by (1) BP neural network training fitting; (2) genetic algorithm extremum seeking. BP neural network training fitting, including the construction of the appropriate neural network, because the function of this example is, so determine the topology of BP network is 2 - 5 - 1. Genetic algorithm extremum seeking, chromosome individuals using real coded, because it is to find the extreme point of the function, so the individual length is 2 . The fitness value of individuals is the predictive value of BP neural network, so the smaller the predicted value is the better the individual. The race was 10 scales, the evolution of 50 times, 0.4 crossover probability, mutation probability 0.2 . BP neural network genetic algorithm optimization extreme, the predictive value of the BP neural network as individual fitness value. Then the optimal genetic algorithm to find the nonlinear function value. 
Scheme Determination. Since the fitness value of individual is the predictive value of BP neural network, the prediction accuracy of BP neural network is of great significance for finding the optimal location. BP neural network prediction output and expected output contrast to the following diagram, the BP neural network can accurately predict the nonlinear function output.

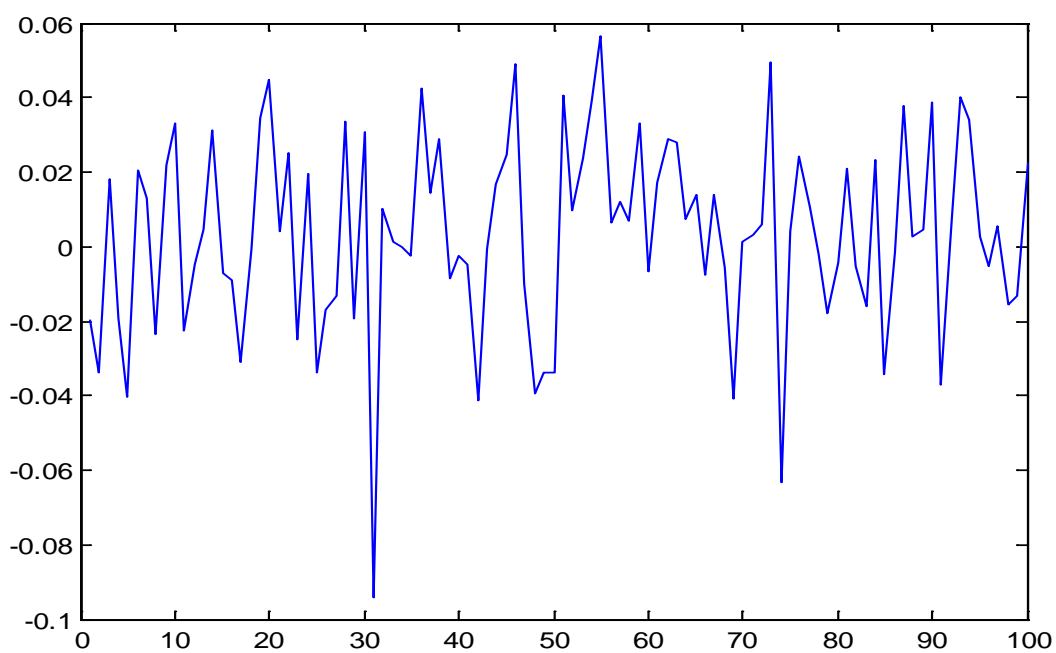

Figure 1. Prediction errors by improved BP neural network optimized by genetic algorithm

\section{Conclusion}

In order to further improve the accuracy of wind power prediction, common on the wind power forecast based on genetic algorithm and BP neural network, genetic algorithm can make up for the shortcomings of BP neural network, such as the initial weights of debugging etc.. Although the neural network algorithm combined with genetic algorithm has improved the prediction accuracy, but there are still some shortcomings in this paper.

\section{References}

[1] Jiang Juan, Ma Xiaoqian, Yu Zhaosheng. Prediction models for incinerator slagging based on BP neural network optimized by genetic algorithm [J]. Renewable Energy Resources, 2010, 28(4): 80-84.

[2] Li Song, Luo Yong, Zhang Mingrui. Prediction method for chaotic time series of optimized BP neural network based on genetic algorithm.Computer Engineering and Applications [J]. Computer Engineering and Applications, 2011, 47(29): 52-55.

[3] Liu Huanyan, Zheng Guanyao,Wang Yanbin, et al. Process parameter optimization of microwave assisted extraction of flavonoids from Ficus carica Linn based on BP neural network [J]. Science and Technology of Food Industry, 2017(6): 1-10.

[4] Zhang Hong, Ma Yan, Li Yong, et al. Rupture energy prediction model for walnut shell breaking based on genetic BP neural network[J]. Transactions of the Chinese Society of Agricultural Engineering (Transactions of the CSAE), 2014, 30(18): 78-84. 